\title{
CAPACITY ESTIMATION OF POLARIZATION-DIVERSITY MIMO SYSTEMS IN URBAN MICROCELLULAR ENVIRONMENTS
}

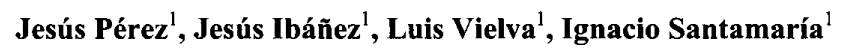 \\ ${ }^{1}$ Universidad de Cantabria, C/Avda. de los Castros S/N, 39005, Santander, Spain, iperez@gtas.dicom.unican.es
}

\begin{abstract}
MIMO systems based on dual-polarized antennas at transmitter and receiver constitute an interesting alternative to conventional MIMO configurations. This paper analyzes the ergodic capacity of such systems in urban micro- and pico-cellular environments. The MIMO channel is modeled by using a site-specific ray-tracing propagation tool. This technique permits to analyze the impact of environmental parameters, like antennas location and orientation, on the system performance. Ergodic capacity estimations in a specific urban environment are presented.
\end{abstract}

Keywords - Microcells, radio propagation, ray tracing, MIMO channels, polarization diversity, channel capacity

\section{INTRODUCTION}

Multiple-input multiple-output (MIMO) systems have revealed as a promising technique to achieve high spectral efficiencies in wireless channels. The conventional approach uses multiple element array antennas at both ends of the wireless link. To exploit the potential benefits of MIMO systems, the spacing between antennas must be large enough, especially in outdoor communications. But in small micro- and pico-cells, the available space uses to be limited. A simple and compact alternative to conventional MIMO systems is to use dual-polarized antennas at transmitter (Tx) and receiver $(\mathrm{Rx})$. In this case, using a single antenna at both ends a $2 \times 2$ MIMO configuration is obtained by employing the two orthogonal polarizations. Recent works have analyzed the potential performance of such systems in terms of capacity [1] or bit error rate (BER) for different coding techniques as Spatial Multiplexing and Space-Time Block coding [2], [3]. These works are based on Rayleigh and Ricean fading MIMO channel models.

In this paper we analyze the ergodic capacity of such MIMO configurations in urban micro- and pico-cellular environments. The channel is simulated by using a sitespecific ray-tracing propagation model [4]. In this way we can estimate the system capacity for specific local areas as well as to analyze how the specific characteristics of the antennas and environment have influence on the system performance.

The paper is organised as follows: In section II we introduce the radio-propagation model. Section III describes the modelling of the MIMO channel from the radio-propagation predictions. The characterization of the system performance, in terms of ergodic capacity, is presented in section IV. Section $V$ shows simulation results in a specific microcellular environment for a specific polarization scheme. Finally, section VI summarizes the conclusions.

\section{PROPAGATION MODEL}

A complete three-dimensional ray-tracing model has been used to predict the field vector at the receiver. The model is based on the Geometric Theory of Diffraction (GTD/UTD) electromagnetic theory, considering specular reflections off ground and buildings as well as diffractions around the buildings wedges. The model requires a detailed description of the environment, which includes geometric and electromagnetic properties of the elements of the propagation scenario: ground and buildings. The ground and buildings walls and roofs are described by planar surfaces indicating their location, shape and orientation. Each surface is also defined by its conductance and electric permittivity. The propagation model also requires as input the location, orientation, polarization and radiation characteristics of the Tx antenna as well as the location of the Rx. During its propagation, each ray can be affected by multiple reflections and diffractions with the surfaces and wedges of the environment. The GTD/UTD model provides the rays reaching the $\mathrm{Rx}$ with the associated E-fields for a given carrier frequency. Detailed information about the propagation model and about other similar models can be found in [4], [5] and [6].

A pure ray-tracing model provides deterministic predictions of the field at the Rx. But the phases of the ray-fields can not be correctly predicted due to the uncertainty of buildings and antennas positions. At frequencies of the order of $\mathrm{GHz}$ 's this uncertainty is always higher than the wavelength. Therefore the phases of the ray-fields are considered as uniformly distributed random variables leading to a socalled SLAC (Stochastic Local Area Channel) model based on the GTD/UTD predictions [7] [8]. This model predicts the statistics of the signal variations due to the multi-path propagation.

Once the GTD/UTD model provides the information about the incoming rays at the receiver, the next step is to calculate the signal induced in the $\mathrm{Rx}$ for each realization of the rays-field. This is calculated by considering the orientation, polarization and radiation characteristics of the $\mathrm{Rx}$ antenna. Since each ray reaches the $\mathrm{Rx}$ from different 
directions, the signals induced by the different rays must be calculated separately.

The polarization and radiation characteristics of the $\mathrm{Rx}$ antenna are defined by the so-called antenna polarization complex vector [9]. As equation (1) shows, this vector is referred to a local spherical coordinate system associated to the antenna. In the particular case of linear-polarized antenna, only one component of the polarization vector is distinct to zero.

$$
\hat{E}^{a}=E_{\theta}^{a} \hat{\theta}_{a}+E_{\phi}^{a} \hat{\phi}_{a}
$$

The signal induced by the $\mathrm{i}$-th ray, can be calculated as the dot product between the E-field of the ray and the antenna polarization vector.

$$
h^{i}=\vec{E}^{i} \cdot \hat{E}^{a}
$$

Note that the E-field of the incoming ray must be previously referred to a local spherical coordinate system. Since the unit vectors of the spherical coordinate depends on the direction of the incoming ray, different rays arriving from different directions contribute to the total received signal in a different manner. Moreover, the local coordinate system depends on the orientation of the Rx antenna, so the antenna orientation will have a significant influence on the induced signals $\left(h^{i}\right)$.

The complex envelope of the channel response will be given by the superposition of the contributions $\left(h^{i}\right)$ of the multipath components [4]. Hence, the model provides realizations of the complex envelope of the channel response taking into account the polarization and radiation characteristics of the antennas.

\section{MIMO CHANNEL MODEL}

The entries of the MIMO channel matrix are the channel response for the corresponding combination of polarizations of the Tx and $\mathrm{Rx}$ antennas. The channel is assumed to be flat over the frequency band and stationary during one symbol interval. Therefore, for a given channell realization, the complex envelope of the received signals can be expressed as follows

$$
\mathbf{r}=\sqrt{\frac{E_{S}}{2}} \mathbf{H} \mathbf{x}+\mathbf{n}
$$

where $\mathbf{r}$ is the $2 \times 1$ received vector, $E_{S}$ is the total average energy transmitted over a symbol interval, $x$ is a $2 x 1$ column vector representing the complex symbols transmitted from a constellation where the average symbol energy is 1 and $\mathbf{n}$ is a $2 \times 1$ vector representing AWGN noise at the receiver where its entries are i.i.d. zero-mean complex Gaussian random variables with variance $\sigma_{\mathrm{n}}{ }^{2} . \mathbf{H}$ is the $2 \times 2$ channel matrix representing a realization of the channel,

$$
\mathbf{H}=\left[\begin{array}{ll}
h_{11} & h_{12} \\
h_{21} & h_{22}
\end{array}\right]
$$

The diagonal elements of $\mathbf{H}$ correspond to transmission and reception on the same polarization, while the off-diagonal entries correspond to transmission and reception on orthogonal polarizations. The entries of $\mathbf{H}$ are obtained from the propagation model as the sum of the contributions of the rays reaching the receiver,

$$
h_{j k}=\sum_{i=1}^{N} h_{j k}^{i}
$$

being $N$ the number of rays and $h_{j k}{ }^{i}$ the contribution of each ray given by (2). As it was mentioned before, the phases of the ray-fields at the receiver are random variables, so the terms $h_{j k}{ }^{i}$ will have random phases and the channel matrix entries will be also random variables. Therefore, the propagation model provides realizations of the MIMO channel matrix.

The potential correlations between the channel matrix entries are implicitly considered by the propagation model. They will depend on the propagation environment, the polarization scheme and the radiation and polarization characteristics of the antennas through the amplitude of the terms $h_{j k}{ }^{i}$.

\section{CHANNEL CAPACITY}

We assume that the channel matrix is known at the $\mathrm{Rx}$, but unknown at the Tx. Then, the ergodic capacity (in bps/ $\mathrm{Hz}$ ) is estimated by averaging the instantaneous capacities over a number of channel realizations.

$$
C=\frac{1}{N} \sum_{i=1}^{N} \log _{2} \operatorname{det}\left(\mathbf{I}_{2}+\frac{E_{S}}{2 \sigma_{n}^{2}} \mathbf{H}_{i} \cdot \mathbf{H}_{i}^{H}\right)
$$

where $\mathbf{I}_{2}$ is the $2 \times 2$ identity matrix, the $\mathbf{H}_{\mathrm{i}}$ are the matrix channel realizations and $\mathrm{N}$ is the number of channel realizations. The subscript $(.)^{H}$ represents the hermitian operator.

Using the GTD/UTD propagation model, the channel prediction includes both the large-scale fading and the small-scale fading. In general, the average capacity will be higher at areas with higher received signal strength. To get further insight into the effects of the small-scale fading, we normalize the channel matrix, removing the average pathloss. The normalization effect is equivalent to perform perfect power control. For each channel realization, the normalized matrix channel will be 


$$
\mathbf{H}_{i}=\frac{1}{\sqrt{L}} \mathbf{H}_{i}^{G T D}
$$

where $\mathbf{H}_{i}{ }^{\text {GTD }}$ is a realization of the MIMO channel matrix given by the propagation model, and $L$ represents the average path-loss. It can be expressed as follows

$$
L=\frac{1}{N} \sum_{i=1}^{N} \frac{\left\|\mathbf{H}_{i}^{G T D}\right\|_{F}^{2}}{4}
$$

$\left\|\mathbf{H}_{\mathrm{i}}{ }^{\mathrm{GTD}}\right\|_{\mathrm{F}}{ }^{2}$ is the squared Frobenius norm of the channel matrix realization given by the propagation model. After this normalization, the average received power at each polarization branch equals the total transmitted power, so the signal-to-noise ratio will be $E_{\mathcal{S}} / \sigma_{n}{ }^{2}$. Hereafter all the capacity values will be obtained from normalized channels.

\section{RESULTS}

The method described above can be used to estimate the ergodic capacity in any micro- or pico-cellular scenario and for any polarization scheme. As an example, figure 1 shows a top view of a micro-cellular scenario consisting of 48 regularly distributed buildings with uniform height $(25 \mathrm{~m})$ and rectangular section, forming a rectilinear grid of parallel and perpendicular streets. The rooftops are assumed to be flat. The area of the environment is $900 \times 500 \mathrm{~m}^{2}$. The Tx antenna is located of $20 \mathrm{~m}$ height on a building wall. The $\mathrm{Rx}$ antenna is always $1.5 \mathrm{~m}$ height.

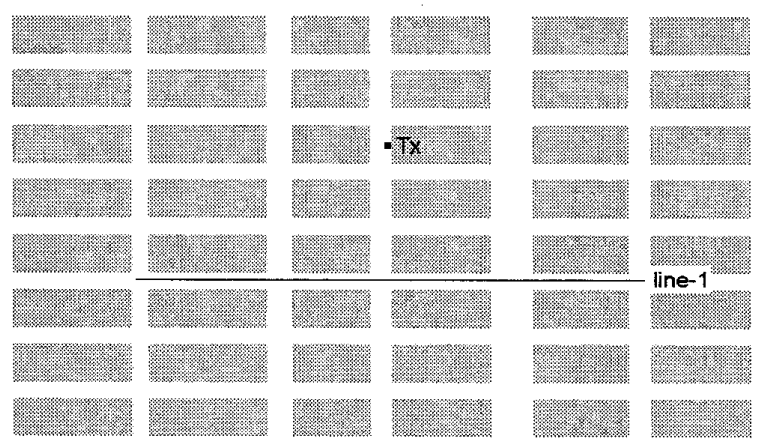

Fig. 1. Top-view of the scenario showing the Tx antenna position. The line shows the $\mathrm{Rx}$ antenna locations in the simulations.

This scenario is an area of the midtown Manhattan which has been used for the validation of propagation models in urban micro-cells [5], [6]. The radio-propagation through the buildings is neglected. We assume that both $\mathrm{Tx}$ and $\mathrm{Rx}$ antennas are omnidirectional in amplitude and phase radiations patters. We also assume that both antennas are dual-linearly polarized and they are vertically oriented.
In all the simulations there were more than 10 rays reaching the $\mathrm{Rx}$ antenna, being the average number of rays around 75 , and 20 in LOS (line-of-sight) and NLOS (non-line-ofsight) points, respectively. All the simulations have been carried out at $1.8 \mathrm{GHz}$. In all the cases the ergodic capacity values correspond to $\mathrm{SNR}=15 \mathrm{~dB}$.

This environment has special propagation characteristics. When the Tx and Rx antennas are below the building roofs, most of the power propagates below the building roofs along the streets. In the other hand, when the Tx antenna is over the building rooftops, most of the power propagates over the buildings up to the street where the $\mathrm{Rx}$ is located.

\section{A. Comparison between LOS and NLOS situations}

Figure 2 shows the simulated ergodic capacity for a fixed $\mathrm{Tx}$ antenna location and variable $\mathrm{Rx}$ position along line 1 (see figure 1). Both, the $\mathrm{Tx}$ and the $\mathrm{Rx}$ antennas have perfect cross-polarization discrimination (XPD $=\infty$ ). As it is expected, the capacity increases significantly when passing from NLOS to LOS points. The curve also shows the significant variations of the capacity at NLOS points as a function of the $\mathrm{Rx}$ antenna location. As reference, the horizontal line shows the ergodic capacity for an uncorrelated Rayleigh MIMO channel.

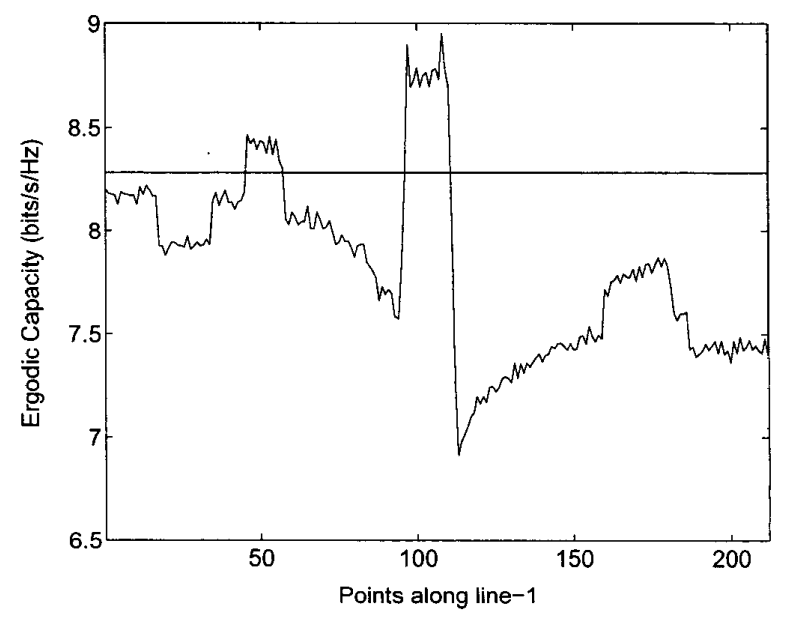

Fig. 2. Ergodic capacity along line-1 of fig. 1.

\section{$B$. Influence of the antennas XPD}

Figure 3 shows the ergodic capacity obtained along line-1 when the TX antenna has XPD $=\infty$ and XPD $=10 \mathrm{~dB}$. As it is expected, higher antenna XPD produces lower correlation in transmission and therefore higher capacity. The XPD introduces a nearly constant shift of about $0.4 \mathrm{bps} / \mathrm{Hz}$ in the ergodic capacity. This behaviour occurs both at LOS and NLOS locations of the receiver. 


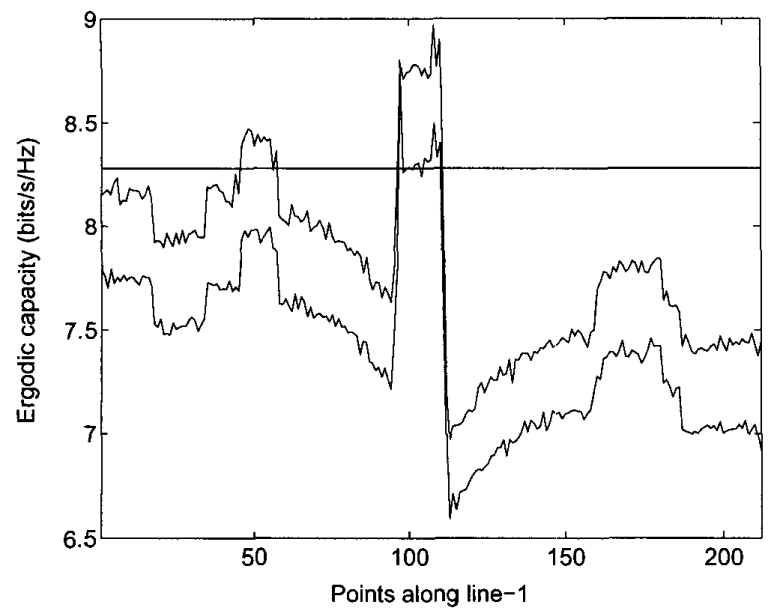

Fig. 3. Ergodic capacity along line- 1 of fig. 1 when the XPD of the Tx antenna is XPD $=\infty$ and XPD $=10 \mathrm{~dB}$.

\section{Capacity along parallel streets}

Figure 4 shows the simulated ergodic capacity along lines parallel to line- 1 but at different streets.

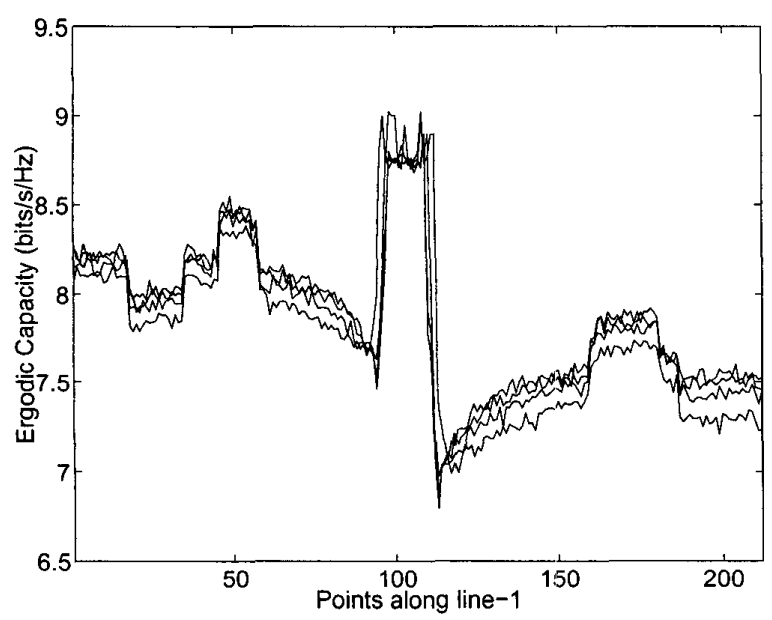

Fig. 4. Ergodic capacity along streets parallel to line-1

The curves are similar because of the symmetry of the environment. No significant variations are observed for different parallel streets. At NLOS points the capacity is slightly lower at streets closer to the Tx antenna. In the other hand, at LOS points slightly higher capacity values are obtained at closer streets to the Tx antenna. In any case, no significant variations are observed between different parallel streets.

\section{Capacity for different Tx antenna heights}

The Tx antenna height does not influence significantly on the capacity values when it is below the building roofs. But it changes meaningfully when the Tx antenna is above the buildings rooftops. In this case new significant propagation paths arise over the buildings. Figure 5 shows the capacity values at the points of line-1 when the Tx antenna is $5 \mathrm{~m}$ below the roofs and when it is $5 \mathrm{~m}$ above the roofs. At LOS points no significant variations are observed. Unlike the LOS case, at NLOS points the capacity changes significantly. In general higher capacity values are obtained when the Tx antenna is above the building rooftops.

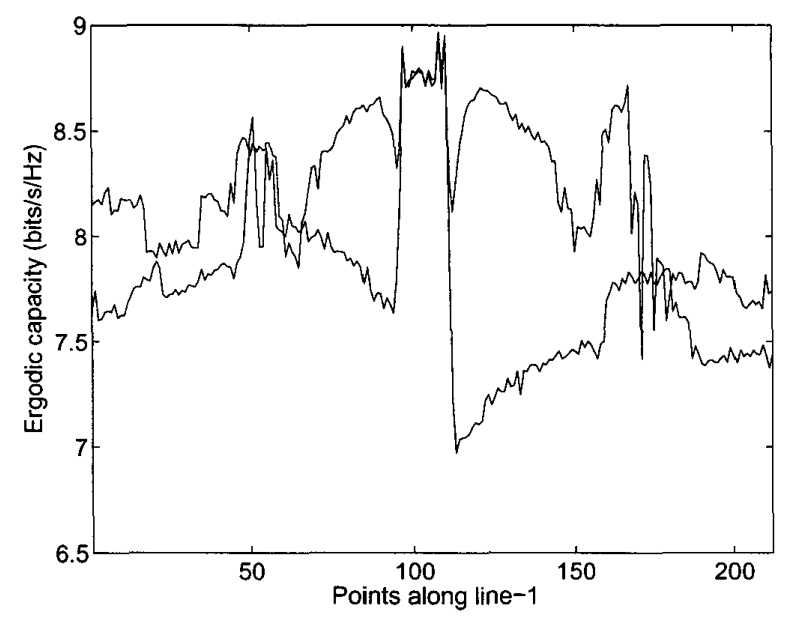

Fig. 5. Ergodic capacity along line- 1 when the Tx antenna is $5 \mathrm{~m}$ below the roofs and $5 \mathrm{~m}$ above the roofs.

\section{CONCLUSIONS}

We have used a site-specific propagation model to estimate the capacity of MIMO systems, based on two dual-polarized antennas, in urban micro-cellular environments. The use of a site-specific propagation model permits us to analyze the capacity variations as function of the scenario, polarization scheme, antennas location, antennas radiation characteristics and antennas polarization characteristics. As an example, we have analyzed the ergodic capacity in a typical urban microcellular scenario characterized by uniform building forming a rectangular grid of parallel and perpendicular streets. The antennas were dual-linear polarized and vertically oriented. We have obtained some conclusions specific of this type of environment. The simulated results show that, in general, the capacity at LOS points is higher than in a NLOS points. The capacity at NLOS points shows significant variations along a street. The XPD of the antennas influences significantly in the capacity values at LOS and NLOS points. In general, higher antenna XPD leads to higher 
capacity values. The capacity values do not change significantly along parallels streets. When the Tx antenna is below the buildings rooftops the capacity values do not change significantly for different heights of the Tx antenna. This is because the propagation characteristics remain nearly constants. In the other hand, when the Tx antenna is above the building, the propagation paths changes significantly and the capacity values also change. It is important to note that some of the above conclusions are specific of this type of propagation environment and can not be generalized to other type of micro-cells. In any case, the method presented can be used to analyze any micro-cellular scenario, any polarization scheme, and any type of antennas. So it permits to study the impact of the environment and antenna characteristics in the capacity of the MIMO system.

\section{ACKNOWLEDGEMENTS}

This work has been partially supported by Spanish Ministry of Science and Technology under project TIC2001-0751C04-03.

\section{REFERENCES}

[1] A. J.Paulraj, R.U.Nabar and D.Gore, Introduction to Space-Time Wireless Communications, Cambridge University Press, 2003.

[2] H.Bölcskei, R.U.Nabar, V.Erceg, D.Gesbert and A. J.Paulraj, "Performance of spatial multiplexing in the presence of polarization diversity", in Proceedings of IEEE ICASSP, Salt Lake City, UT, vol. 4, pp. 24372440, May 2001.

[3] R.U.Nabar, H.Bölcskei, V.Erceg, D.Gesbert and A. J.Paulraj, "Performance of Multiantenna Signaling Techniques in the Presence of Polarization Diversity", IEEE Transactions on Signal Processing, vol. 50, pp. 2553-2562, October 2002.

[4] M.F.Cátedra and J.Pérez, Cell Planning for wireless Communications, Artech House, Norwood, MA, 1999.

[5] S.Y.Tan and H.S.Tan, "A Microcellular Communications Propagation Model Based on the Uniform Theory of Diffraction and Multiple Image Theory", IEEE Transactions on Antennas and Propagation, vol. 44, pp. 1317-1326, October 1996.

[6] V.Erceg, S.J.Fortune, L.Ling, A.J.Rustako and R.A.Valenzuela, "Comparison of Computer-Based Propagation Prediction Tool with Experimental Data Collected in Urban Microcellular Environments", IEEE Journal on Selected Areas in Communications, vol. 15, pp. 677-684, May 1997.

[7] G.D.Durgin, Space-Time Wireless Channels, Prentice Hall PTR, Upper Saddle River, NJ, 2003.

[8] H. Zhu, J.Takada, K.Araki and T.Kobayashi, "A random-phase-assisted ray-tracing code for wireless channel modeling", Applied Computational
Electromagnetic Society Journal, vol. XVI, pp. 69-78, March 2001.

[9] C.A.Balanis, Antenna Theory. Analysis and Design, John Wiley and Sons, New York, 1997. 